Paper solicited for the Special Session: Resonant BAW sensors and actuators

\title{
Resonant Ultrasonic Particle Manipulators and their Applications in Sensor Systems
}

\author{
Martyn Hill, Jeremy J Hawkes ${ }^{*}$, Nick R Harris ${ }^{+}$ \\ School of Engineering Sciences, University of Southampton, UK, m.hill@soton.ac.uk \\ *Department of Instrumentation and Analytical Science, UMIST, Manchester, UK, \\ ${ }^{+}$Electronics and Computer Science, University of Southampton, UK,
}

\section{Summary}

This paper describes the use of ultrasonic standing waves as bulk acoustic wave actuators, exploiting the acoustic radiation forces within the standing wave to move biological cells or other particles. This is a new mechanism to enhance many forms of microflow based sensors. Example applications: half-wavelength sample-preparation chambers, move cells from one fluid medium into another (washing the cells) and quarter wavelength chambers force cells up against a solid boundary such as the face of a sensor. Models and tests are used to describe these resonators which contain many, subwavelength resonances, and the creation of the next generation multilayer thick films pzt actuatation for driving these microfluidic particle manipulators.

\section{Motivation}

The development of sensors in microflow-systems introduces new handling needs, particularly to remove particle contaminants and to enhance particle-detection at a sensor surface. In these low Reynolds number systems particle manipulation is dominated by laminar flow; thermal convection is minimal, electrical forces are inconsistent in the variable conductivity environments of medical and microbiological cell suspensions. Acoustic radiation forces however have been shown to be highly suitable for separating biological cells in microflow-systems (sub-wavelength chambers) ${ }^{1}$.

\section{Results}

An example of the type of layered resonator used to achieve this is shown in the $3 \mathrm{MHz}$ microfabricated example of fig. 1. Modelling and testing of resonators produced systems designed to move cells to either the centre or boundary of a chamber depending on the operating frequency (fig. 2). In an application where spores are captured on an antibody coated surface the sound in a quarter-wave chamber forces the spores up against a solid boundary resulting in a 200 fold increase in capture rate (fig. 3). In the washing application, the medium surrounding yeast cells has been reduced 6 fold (figs 4 \& 5). Developments to replace the bulk pzt in such resonators are currently able to form a multilayer thick film pzt actuator which can generate acoustic power very efficiently at around $4 \mathrm{MHz}$ (fig. 6).

\footnotetext{
${ }^{1}$ Hawkes, J.J., Coakley, W.T. "Force field particle filter, combining ultrasound standing waves and laminar flow”. Sens. Actuators B, vol 75.,p. 213-222, 2001.
} 


\section{Figures}

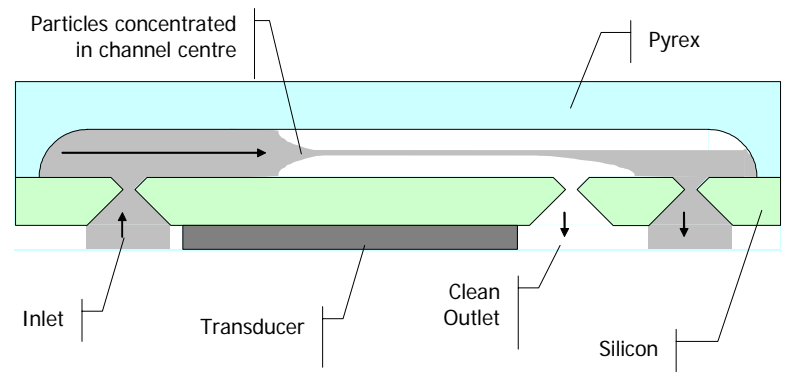

Fig. 1 Schematic representation of a silicon/Pyrex ultrasonic microfluidic filter (not to scale).

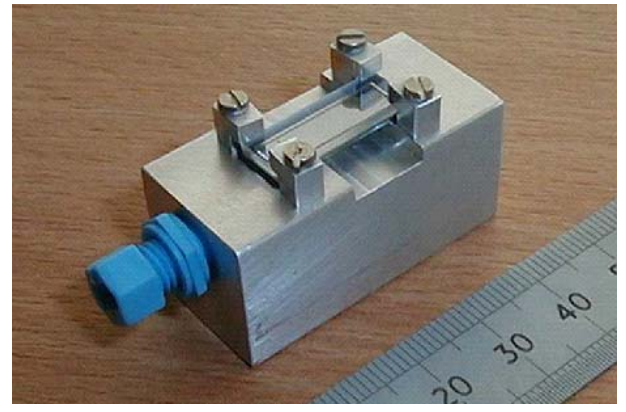

Fig. 2. A micromachined resonator (shown in fig 1) on an aluminium manifold. The resonator allows particles to be moved to either boundary of the fluid layer, or to the centre of the layer, depending on the driving frequency.

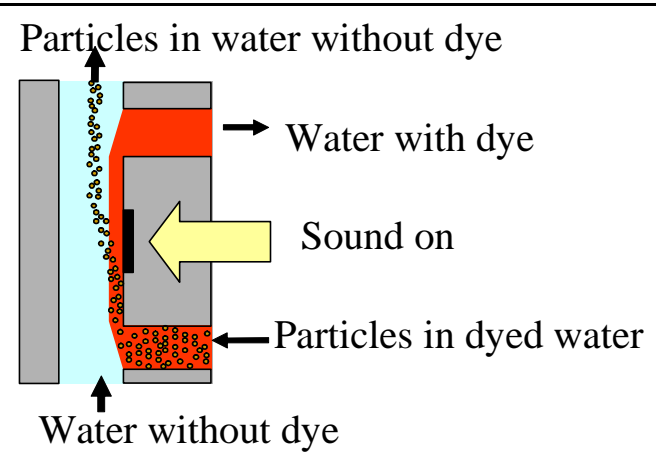

Fig. 4. Schematic representation of ultrasonic particle washing chamber.
Fig. 3. Spore adhesion to antibody coated glass across the boundary of the acoustically excited region, showing significant spore concentration within the acoustic field on the right of the image.

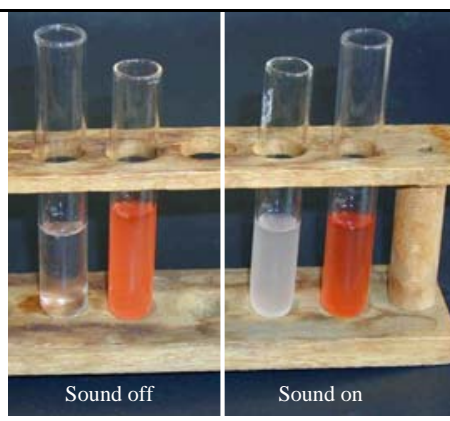

Fig. 5. Fluid collected from outlet streams of fig. 4. The turbidity of the samples shows that the particles remain in the dyed water without ultrasound, but move into the water without dye when the ultrasound is turned on.

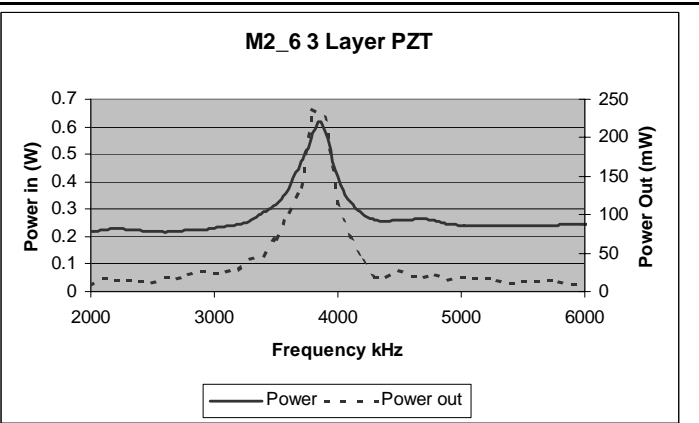

Fig. 6. Power measurements for a three layer pzt actuator driven at around 4 $\mathrm{MHz}$ 